\title{
On the SQH Method for Solving Differential Nash Games
}

\author{
Francesca Calà Campana ${ }^{1}$ - Alfio Borzi ${ }^{1}$ iD
}

Received: 3 January 2021 / Revised: 22 March 2021 / Accepted: 27 March 2021 /

Published online: 27 April 2021

(C) The Author(s) 2021

\begin{abstract}
A sequential quadratic Hamiltonian scheme for solving open-loop differential Nash games is proposed and investigated. This method is formulated in the framework of the Pontryagin maximum principle and represents an efficient and robust extension of the successive approximations strategy for solving optimal control problems. Theoretical results are presented that prove the well-posedness of the proposed scheme, and results of numerical experiments are reported that successfully validate its computational performance.
\end{abstract}

Keywords Sequential quadratic Hamiltonian method · Differential Nash games · Pontryagin maximum principle $\cdot$ Successive approximations strategy

Mathematics Subject Classification (2010) 49K15 · 49N70 · 49M05 - 65K10 · 91A10

\section{Introduction}

The sequential quadratic Hamiltonian (SQH) scheme has been recently proposed in [4-7] for solving nonsmooth optimal control problems governed by differential models. The SQH scheme belongs to the class of iterative methods known as successive approximations (SA) schemes that are based on the characterisation of optimality in control problems by the Pontryagin maximum principle (PMP); see [2, 27] and [12] for a recent detailed discussion. The initial development of SA schemes was inspired by the work of L. I. Rozonoèr [32], and originally proposed in different variants by H.J. Kelley, R.E. Kopp and H.G. Moyer [19] and by I.A. Krylov and F.L. Chernous'ko [20, 21]; see [11] for an early review.

The working principle of most SA schemes is the point-wise minimisation of the Hamilton-Pontryagin function introduced in the PMP theory. However, in their original formulation, the SA schemes appeared efficient but not robust with respect to the numerical

Alfio Borzì

alfio.borzi@mathematik.uni-wuerzburg.de

Francesca Calà Campana

francesca.cala-campana@mathematik.uni-wuerzburg.de

1 Institut für Mathematik, Universität Würzburg, Emil-Fischer-Strasse 30, Würzburg, 97074, Germany 
and optimisation parameters. Twenty years later, a great improvement in robustness was achieved by Y. Sakawa and Y. Shindo $[33,34]$ by introducing a quadratic penalty of the control updates that resulted in an augmented Hamiltonian. In this latter formulation, the need of coupled updates of the state and control variables of the controlled system limited the application of the resulting method to small-size control problems. This limitation was resolved in the SQH approach where a sequential point-wise optimisation of an augmented Hamiltonian function is considered that defines a suitable update step for the control variable while the state function is updated after the completion of this step. Since the SQH iterative procedure has proved efficient and robust in solving (non-smooth) optimal control problems governed by ordinary- and partial-differential models, it is reasonable to investigate whether this procedure can be successfully extended to differential games.

The first component of a differential game is a differential model that governs the state of the considered system and is subject to different mechanisms of action representing the strategies of the players in the game. Furthermore, objective functionals and admissible set of actions are associated to each player, and in the game the purpose of each player is to minimise its own objective subject to the constraints given by the differential model and the admissible sets. Since we consider non-cooperative games, a suitable solution concept is the one proposed by J. F. Nash in [22, 23], where a so-called Nash equilibrium (NE) for static games with complete information is defined, that is, a configuration where no player can benefit from unilaterally changing its own strategy. In this framework, differential Nash games were pioneered by R. P. Isaacs [16]. However, contemporary to Isaacs' book, there are the works $[25,26]$ where differential games are discussed in the framework of the maximum principle. Furthermore, in [1, 21], we find early attempts and comments towards the development of a SA scheme for differential games. Unfortunately, less attention was paid to this research direction and the further development of these schemes for differential games was left out.

It is the purpose of this work to contribute to this development by investigating a SQH scheme for solving open-loop non-zero-sum two-player differential Nash games. In particular, we consider linear-quadratic (LQ) Nash games that appear in the field of, e.g., economics and marketing $[13,18]$, and are very well investigated from the theoretical point of view; see, e.g., [8, 14, 15, 35]. Moreover, since the solution of unconstrained LQ Nash games can be readily obtained by solving coupled Riccati equations [3, 14], they provide a convenient benchmark for our method. However, we also consider extension of LQ Nash games to problems with tracking objectives, box constraints on the players' actions, and actions' costs that include $L^{1}$ terms.

In the next section, we formulate a class of differential Nash games and their characterisation in the PMP framework. In particular, we notice that the point-wise PMP characterisation of a Nash equilibrium corresponds to the requirement that, at each time instant, the conditions of equilibrium of a finite-dimensional Nash game with two HamiltonPontryagin functions must be satisfied. In Section 3, we present our SQH method for solving differential Nash games and discuss its well-posedness. Specifically, we show that the adaptive choice of the weight of a Sakawa-Shindo-type penalisation can be successfully performed in a finite number of steps such that the proposed update criteria based on the Nikaido-Isoda function are satisfied.

Section 4 is devoted to numerical experiments that successfully validate our computational framework. In the first experiment, we consider a differential LQ Nash game and show that the SQH scheme provides a solution that is identical to that obtained by solving an appropriate Riccati system. In the second experiment, the same problem with the addition of the requirement that the players' actions must belong to given bounded, closed, and convex 
sets is solved by the SQH scheme. In the third experiment, we extend the setting of the second experiment by adding weighted $L^{1}$ costs of the players' actions and verify that these costs promote sparsity. In the fourth experiment, we consider the case where each player's functional corresponds to a tracking problem where the players aim at following different paths. Also in this case, constraints on the players' actions and $L^{1}$ costs are considered. We remark that all NE solutions are successfully computed with the same setting of values of the parameters entered in the SQH algorithm, that is, independently of the problem and of the chosen weights in the players' cost functionals. A section of conclusion completes this work.

\section{PMP Characterization of Nash Games}

This section is devoted to the formulation of differential Nash games and the characterisation of their NE solution in the PMP framework. We discuss the case of two players, represented by their strategies $\underline{u}_{1}$ and $\underline{u}_{2}$, which can be readily extended to the case of $N$ players, and assume the following dynamics:

$$
\underline{y}^{\prime}(t)=\underline{f}\left(t, \underline{y}(t), \underline{u}_{1}(t), \underline{u}_{2}(t)\right), \quad \underline{y}(0)=\underline{y}_{0},
$$

where $t \in[0, T], y(t) \in \mathbb{R}^{n}$, and $\underline{u}_{1}(t) \in \mathbb{R}^{m}$ and $\underline{u}_{2}(t) \in \mathbb{R}^{m}, m \leq n$. We assume that $f$ is such that for any choice of the initial condition $\underline{y}_{0} \in \mathbb{R}^{n}$, and any $\underline{u}_{1}, \underline{u}_{2} \in L^{2}\left(0, T ; \overline{\mathbb{R}}^{m}\right)$, the Cauchy problem (1) admits a unique solution in the sense of Carathéodory; see, e.g., [3]. Furthermore, we assume that the map $\left(\underline{u}_{1}, \underline{u}_{2}\right) \mapsto \underline{y}=\underline{y}\left(\underline{u}_{1}, \underline{u}_{2}\right)$, where $\underline{y}\left(\underline{u}_{1}, \underline{u}_{2}\right)$ represents the unique solution to Eq. 1 with fixed initial conditions is continuous in $\left(\underline{u}_{1}, \underline{u}_{2}\right)$.

We refer to $\underline{u}_{1}$ and $\underline{u}_{2}$ as the game strategies of the players $P_{1}$ and $P_{2}$, respectively. The goal of $P_{1}$ is to minimise the following cost (or objective) functional:

$$
J_{1}\left(\underline{y}, \underline{u}_{1}, \underline{u}_{2}\right):=\int_{0}^{T} \ell_{1}\left(t, \underline{y}(t), \underline{u}_{1}(t), \underline{u}_{2}(t)\right) d t+g_{1}(\underline{y}(T)),
$$

whereas $P_{2}$ aims at minimising its own objective given by

$$
J_{2}\left(\underline{y}, \underline{u}_{1}, \underline{u}_{2}\right):=\int_{0}^{T} \ell_{2}\left(t, \underline{y}(t), \underline{u}_{1}(t), \underline{u}_{2}(t)\right) d t+g_{2}(\underline{y}(T)) .
$$

We consider the cases of unconstrained and constrained strategies. In the former case, we assume $\underline{u}_{1}, \underline{u}_{2} \in L^{2}\left(0, T ; \mathbb{R}^{m}\right)$, whereas in the latter case we assume that $\underline{u}_{1}$ and $\underline{u}_{2}$ belong, respectively, to the following admissible sets:

$$
U_{a d}^{(i)}=\left\{\underline{u} \in L^{2}\left(0, T ; \mathbb{R}^{m}\right): \underline{u}(t) \in K_{a d}^{(i)}, t \in[0, T]\right\}, \quad i=1,2,
$$

where $K_{a d}^{(i)}$ are compact and convex subsets of $\mathbb{R}^{m}$. We denote with $U_{a d}=U_{a d}^{(1)} \times U_{a d}^{(2)}$ and $U=L^{2}\left(0, T ; \mathbb{R}^{m}\right) \times L^{2}\left(0, T ; \mathbb{R}^{m}\right)$. Notice that we have a uniform bound on $\left|\underline{y}\left(\underline{u}_{1}, \underline{u}_{2}\right)(t)\right|$, $t \in[0, T]$, that holds for any $\underline{u} \in U_{a d}$; see [9].

By using the map $\left(\underline{u}_{1}, \underline{u}_{2}\right) \mapsto \underline{y}=\underline{y}\left(\underline{u}_{1}, \underline{u}_{2}\right)$, we can introduce the reduced objectives $\tilde{J}_{1}\left(\underline{u}_{1}, \underline{u}_{2}\right):=J_{1}\left(\underline{y}\left(\underline{u}_{1}, \underline{u}_{2}\right), \underline{u}_{1}, \underline{u}_{2}\right)$ and $\tilde{J}_{2}\left(\underline{u}_{1}, \underline{u}_{2}\right):=J_{2}\left(\underline{y}\left(\underline{u}_{1}, \underline{u}_{2}\right), \underline{u}_{1}, \underline{u}_{2}\right)$. In this framework, a Nash equilibrium is defined as follows. 
Definition 1 The functions $\left(\underline{u}_{1}^{*}, \underline{u}_{2}^{*}\right) \in U_{a d}$ are said to form a Nash equilibrium (NE) for the game $\left(\tilde{J}_{1}, \tilde{J}_{2} ; U_{a d}^{(1)}, U_{a d}^{(2)}\right)$, if it holds

$$
\begin{array}{ll}
\tilde{J}_{1}\left(\underline{u}_{1}^{*}, \underline{u}_{2}^{*}\right) \leq \tilde{J}_{1}\left(\underline{u}_{1}, \underline{u}_{2}^{*}\right), & \underline{u}_{1} \in U_{a d}^{(1)}, \\
\tilde{J}_{2}\left(\underline{u}_{1}^{*}, \underline{u}_{2}^{*}\right) \leq \tilde{J}_{2}\left(\underline{u}_{1}^{*}, \underline{u}_{2}\right), & \underline{u}_{2} \in U_{a d}^{(2)} .
\end{array}
$$

(A similar Nash game is defined replacing $U_{a d}$ with $U$.)

We remark that existence of a NE point can be proved subject to appropriate conditions on the structure of the differential game, including the choice of $T$. For our purpose, we assume existence of a Nash equilibrium $\left(\underline{u}_{1}^{*}, \underline{u}_{2}^{*}\right) \in U_{a d}$, and refer to [9] for a review and recent results in this field.

We remark that, if $\underline{u}^{*}=\left(\underline{u}_{1}^{*}, \underline{u}_{2}^{*}\right)$ is a NE for the game, then it satisfies the following:

$$
\underline{u}_{1}^{*}=\underset{\underline{u}_{1} \in U_{a d}^{(1)}}{\arg \min } \tilde{J}_{1}\left(\underline{u}_{1}, \underline{u}_{2}^{*}\right), \quad \underline{u}_{2}^{*}=\underset{\underline{u}_{2} \in U_{a d}^{(2)}}{\arg \min } \tilde{J}_{2}\left(\underline{u}_{1}^{*}, \underline{u}_{2}\right) .
$$

This fact implies that the NE point $\underline{u}^{*}=\left(\underline{u}_{1}^{*}, \underline{u}_{2}^{*}\right)$ must fulfil the necessary optimality conditions given by the Pontryagin maximum principle applied to both optimisation problems stated in Eq. 5, alternatively (6).

In order to discuss these conditions, we introduce the following Hamilton-Pontryagin (HP) functions:

$$
\mathcal{H}_{i}\left(t, \underline{y}, \underline{u}_{1}, \underline{u}_{2}, \underline{p}_{1}, \underline{p}_{2}\right)=\underline{p}_{i} \cdot \underline{f}\left(t, \underline{y}, \underline{u}_{1}, \underline{u}_{2}\right)-\ell_{i}\left(t, \underline{y}, \underline{u}_{1}, \underline{u}_{2}\right), \quad i=1,2 .
$$

In terms of these functions, the PMP condition for the NE point $\underline{u}^{*}=\left(\underline{u}_{1}^{*}, \underline{u}_{2}^{*}\right)$ states the existence of multiplier (adjoint) functions $\underline{p}_{1}, \underline{p}_{2}:[0, T] \rightarrow \mathbb{R}^{n}$ such that the following holds:

$$
\begin{gathered}
\max _{\underline{w}_{1} \in K_{a d}^{(1)}} \mathcal{H}_{1}\left(t, \underline{y}^{*}(t), \underline{w}_{1}, \underline{u}_{2}^{*}(t), \underline{p}_{1}^{*}(t), \underline{p}_{2}^{*}(t)\right)=\mathcal{H}_{1}\left(t, \underline{y}^{*}(t), \underline{u}_{1}^{*}(t), \underline{u}_{2}^{*}(t), \underline{p}_{1}^{*}(t), \underline{p}_{2}^{*}(t)\right), \\
\quad \max _{\underline{w}_{2} \in K_{a d}^{(2)}}
\end{gathered}
$$

for almost all $t \in[0, T]$. Notice that, at each $t$ fixed, the problem (8) corresponds to a finite-dimensional Nash game.

In Eq. 8, we have $\underline{y}^{*}=y\left(\underline{u}_{1}^{*}, \underline{u}_{2}^{*}\right)$, and the adjoint variables $\underline{p}_{1}^{*}, \underline{p}_{2}^{*}$ are the solutions to the following differential problems:

$$
\begin{aligned}
-\underline{p}_{i}^{\prime}(t) & =\left(\partial_{y} \underline{f}\left(t, \underline{y}(t), \underline{u}_{1}(t), \underline{u}_{2}(t)\right)\right)^{\top} \underline{p}_{i}(t)-\partial_{y} \ell_{i}\left(t, \underline{y}(t), \underline{u}_{1}(t), \underline{u}_{2}(t)\right), \\
\underline{p}_{i}(T) & =-\partial_{y} g_{i}(\underline{y}(T)),
\end{aligned}
$$

where $i=1,2, \partial_{y} \phi(y)$ represents the Jacobian of $\phi$ with respect to the vector of variables $y$, and $\top$ means transpose. Similarly to Eq. 1, one can prove that Eqs. 9 and 10 are uniquely solvable, and the solution can be uniformly bounded independently of $\underline{u} \in U_{a d}$.

We conclude this section introducing the Nikaido-Isoda [24] function $\psi: U_{a d} \times U_{a d} \rightarrow$ $\mathbb{R}$, which we use for the realisation of the SQH algorithm. We have

$$
\psi(\underline{u}, \underline{v}):=\tilde{J}_{1}\left(\underline{u}_{1}, \underline{u}_{2}\right)-\tilde{J}_{1}\left(\underline{v}_{1}, \underline{u}_{2}\right)+\tilde{J}_{2}\left(\underline{u}_{1}, \underline{u}_{2}\right)-\tilde{J}_{2}\left(\underline{u}_{1}, \underline{v}_{2}\right),
$$


where $\underline{u}=\left(\underline{u}_{1}, \underline{u}_{2}\right) \in U_{a d}$ and $\underline{v}=\left(\underline{v}_{1}, \underline{v}_{2}\right) \in U_{a d}$. At the Nash equilibrium $\underline{u}^{*}=\left(u_{1}^{*}, u_{2}^{*}\right)$ it holds

$$
\psi\left(\underline{u}^{*}, \underline{v}\right) \leq 0
$$

for any $\underline{v} \in U_{a d}$ and $\psi\left(\underline{u}^{*}, \underline{u}^{*}\right)=0$.

\section{The SQH Scheme for Solving Nash Games}

In the spirit of the SA scheme proposed by Krylov and Chernous'ko [20], a SA methodology for solving our Nash game $\left(\tilde{J}_{1}, \tilde{J}_{2} ; U_{a d}^{(1)}, U_{a d}^{(2)}\right)$ consists of an iterative process, starting with an initial guess $\left(\underline{u}_{1}^{0}, \underline{u}_{2}^{0}\right) \in U_{a d}$, and followed by the solution of our governing model (1) and of the adjoint problems Eqs. 9 and 10 for $i=1,2$. Thereafter, a new approximation to the strategies $\underline{u}_{1}$ and $\underline{u}_{2}$ is obtained by solving, at each $t$ fixed, the Nash game (8) and assigning the values of $\left(\underline{u}_{1}(t), \underline{u}_{2}(t)\right)$ equal to the solution of this game.

We remark that this update step is well posed if this solution exists for $t \in[0, T]$ and the resulting functions $\underline{u}_{1}$ and $\underline{u}_{2}$ are measurable. Clearly, this issue requires identifying classes of problems for which we can guarantee existence and uniqueness (or the possibility of selection) of a NE point. In this respect, a large class can be identified based on the following result given in [8], which is proved by an application of the Kakutani's fixed-point theorem; see, e.g., [3] for references. We have

Theorem 3.1 Assume the following structure:

$$
\underline{f}\left(t, \underline{y}, \underline{u}_{1}, \underline{u}_{2}\right)=\underline{f}_{0}(t, \underline{y})+M_{1}(t, \underline{y}) \underline{u}_{1}+M_{2}(t, \underline{y}) \underline{u}_{2},
$$

and

$$
\ell_{i}\left(t, \underline{y}, \underline{u}_{1}, \underline{u}_{2}\right)=\ell_{i}^{0}(t, \underline{y})+\ell_{i}^{1}\left(t, \underline{u}_{1}\right)+\ell_{i}^{2}\left(t, \underline{u}_{2}\right), \quad i=1,2 .
$$

Furthermore, suppose that $K_{a d}^{(1)}$ and $K_{a d}^{(2)}$ are compact and convex, the function $\underline{f}_{0}, \ell_{i}^{0}$ and the matrix functions $M_{1}$ and $M_{2}$ are continuous in $t$ and $\underline{y}$, and the functions $\underline{u}_{1} \rightarrow \ell_{1}^{1}\left(t, \underline{u}_{1}\right)$ and $\underline{u}_{2} \rightarrow \ell_{2}^{2}\left(t, \underline{u}_{2}\right)$ are strictly convex for any choice of $t \in[0, T]$ and $\underline{y} \in \mathbb{R}^{n}$.

Then, for any $t \in[0, T]$ and any $\underline{y}, \underline{p}_{1}, \underline{p}_{2} \in \mathbb{R}^{n}$, there exists a unique pair $\left(\underline{\tilde{u}}_{1}, \underline{\tilde{u}}_{2}\right) \in$ $K_{a d}^{(1)} \times K_{a d}^{(2)}$ such that

$$
\begin{gathered}
\tilde{u}_{1}=\underset{\underline{v} \in K_{a d}^{(1)}}{\arg \max }\left(\underline{p}_{1} \cdot \underline{f}\left(t, \underline{y}, \underline{v}, \underline{\tilde{u}}_{2}\right)-\ell_{1}\left(t, \underline{y}, \underline{v}, \underline{\tilde{u}}_{2}\right)\right), \\
\tilde{u}_{2}=\underset{\underline{w} \in K_{a d}^{(2)}}{\arg \max }\left(\underline{p}_{2} \cdot \underline{f}\left(t, \underline{y}, \underline{\tilde{u}}_{1}, \underline{w}\right)-\ell_{2}\left(t, \underline{y}, \underline{\tilde{u}}_{1}, \underline{w}\right)\right) .
\end{gathered}
$$

With the setting of this theorem, the map $\left(t, \underline{y}, \underline{p}_{1}, \underline{p}_{2}\right) \mapsto\left(u_{1}^{*}, u_{2}^{*}\right)$ is continuous [8]. Moreover, based on results given in [30], one can prove that the functions $\left(\underline{u}_{1}(t), \underline{u}_{2}(t)\right)$ resulting from the SA update, starting from measurable $\left(\underline{u}_{1}^{0}(t), \underline{u}_{2}^{0}(t)\right)$, are measurable. Therefore, the proposed SA update is well-posed and it can be repeated in order to construct a sequence of functions $\left(\left(\underline{u}_{1}^{k}, \underline{u}_{2}^{k}\right)\right)_{k=0}^{\infty}$.

However, as already pointed out in [20] in the case of optimal control problems, it is difficult to find conditions that guarantee convergence of SA iterations to the solution sought. Furthermore, results of numerical experiments show a lack of robustness of the SA 
scheme with respect to the choice of the initial guess and of the numerical and optimisation parameters.

For this reason, further research effort was put in the development of the SA strategy, and an advancement was achieved by Sakawa and Shindo considering a quadratic penalty on the Hamiltonian $[33,34]$. We remark that these authors related their penalisation strategy to that proposed by B. Järmark in [17], which is similar to the proximal scheme of R. T. Rockafellar discussed in [29].

For our purpose, we follow the same path of [33] and extend it to the case of Nash games as follows. Consider the following augmented HP functions:

$$
\mathcal{K}_{\epsilon}^{(i)}\left(t, \underline{y}, \underline{u}_{1}, \underline{u}_{2}, \underline{v}_{1}, \underline{v}_{2}, \underline{p}_{1}, \underline{p}_{2}\right):=\mathcal{H}_{i}\left(t, \underline{y}, \underline{u}_{1}, \underline{u}_{2}, \underline{p}_{1}, \underline{p}_{2}\right)-\epsilon|\underline{u}-\underline{v}|^{2}, \quad i=1,2,
$$

where, in the iteration process, $\underline{u}=\left(\underline{u}_{1}, \underline{u}_{2}\right)$ is subject to the update step, and $\underline{v}=\left(\underline{v}_{1}, \underline{v}_{2}\right)$ corresponds to the previous strategy approximation; $|\cdot|$ denotes the Euclidean norm. The parameter $\epsilon>0$ represents the augmentation weight that is chosen adaptively along the iteration as discussed below.

Now, similar to the SA update illustrated above, suppose that the $k$ th function approximation $\left(\underline{u}_{1}^{k}, \underline{u}_{2}^{k}\right)$ and the corresponding $\underline{y}^{k}$ and $\underline{p}_{1}^{k}, \underline{p}_{2}^{k}$ have been computed. For any fixed $t \in[0, T]$ and $\epsilon>0$, consider the following finite-dimensional Nash game:

$$
\begin{aligned}
& \mathcal{K}_{\epsilon}^{(1)}\left(t, \underline{y}^{k}, \underline{\tilde{u}}_{1}, \underline{\tilde{u}}_{2}, \underline{u}_{1}^{k}, \underline{u}_{2}^{k}, \underline{p}_{1}^{k}, \underline{p}_{2}^{k}\right)=\max _{\underline{u}_{1} \in K_{a d}^{(1)}} \mathcal{K}_{\epsilon}^{(1)}\left(t, \underline{y}^{k}, \underline{u}_{1}, \underline{\tilde{u}}_{2}, \underline{u}_{1}^{k}, \underline{u}_{2}^{k}, \underline{p}_{1}^{k}, \underline{p}_{2}^{k}\right), \\
& \mathcal{K}_{\epsilon}^{(2)}\left(t, \underline{y}^{k}, \underline{\tilde{u}}_{1}, \underline{\tilde{u}}_{2}, \underline{u}_{1}^{k}, \underline{u}_{2}^{k}, \underline{p}_{1}^{k}, \underline{p}_{2}^{k}\right)=\max _{\underline{u}_{2} \in K_{a d}^{(2)}} \mathcal{K}_{\epsilon}^{(2)}\left(t, \underline{y}^{k}, \underline{\tilde{u}}_{1}, \underline{u}_{2}, \underline{u}_{1}^{k}, \underline{u}_{2}^{k}, \underline{p}_{1}^{k}, \underline{p}_{2}^{k}\right),
\end{aligned}
$$

where $\underline{y}^{k}=\underline{y}^{k}(t), \underline{p}_{1}^{k}=\underline{p}_{1}^{k}(t), \underline{p}_{2}^{k}=\underline{p}_{2}^{k}(t)$, and $\left(\underline{u}_{1}^{k}, \underline{u}_{2}^{k}\right)=\left(\underline{u}_{1}^{k}(t), \underline{u}_{2}^{k}(t)\right)$.

It is clear that, assuming the structure specified in Theorem 3.1, the Nash game (14) admits a unique NE point, $\left(\underline{\tilde{u}}_{1}, \underline{\tilde{u}}_{2}\right) \in K_{a d}^{(1)} \times K_{a d}^{(2)}$, and the sequence constructed recursively by the procedure:

$$
\left(\underline{u}_{1}^{k}(t), \underline{u}_{2}^{k}(t)\right) \rightarrow\left(\underline{u}_{1}^{k+1}(t), \underline{u}_{2}^{k+1}(t)\right)=\left(\underline{\tilde{u}}_{1}, \underline{\tilde{u}}_{2}\right)
$$

is well defined.

Notice that, in this procedure, the solution to Eq. 14 depends on the value of $\epsilon$. Therefore, the issue arises whether, corresponding to the step $k \rightarrow k+1$, we can choose the value of this parameter such that the strategy function $\underline{u}^{k+1}=\left(\underline{u}_{1}^{k+1}, \underline{u}_{2}^{k+1}\right)$ represents an improvement on $\underline{u}^{k}=\left(\underline{u}_{1}^{k}, \underline{u}_{2}^{k}\right)$, in the sense that some convergence criteria towards the solution to our differential Nash problem are fulfilled.

For this purpose, we define a criterion that is based on the Nikaido-Isoda function. We require that

$$
\psi\left(\underline{u}^{k+1}, \underline{u}^{k}\right) \leq-\xi\left\|\underline{u}^{k+1}-\underline{u}^{k}\right\|_{L^{2}\left(0, T ; \mathbb{R}^{m}\right)}^{2},
$$

for some chosen $\xi>0$. This is a consistency criterion in the sense that $\psi$ must be nonpositive, and if $\left(\underline{u}^{k+1}, \underline{u}^{k}\right) \rightarrow\left(\underline{u}^{*}, \underline{u}^{*}\right)$, then we must have $\lim _{k \rightarrow \infty} \psi\left(\underline{u}^{k+1}, \underline{u}^{k}\right)=0$. Furthermore, we require that the absolute value $\left|\psi\left(\underline{u}^{k+1}, \underline{u}^{k}\right)\right|$ monotonically decreases in the $\mathrm{SQH}$ iteration process.

In our SQH scheme, if the strategy update meets the two requirements above, then the update is taken and the value of $\epsilon$ is diminished by a factor $\zeta \in(0,1)$. If not, the update is discarded and the value of $\epsilon$ is increased by a factor $\sigma>1$, and the procedure is repeated. 
Below, we show that a value of $\epsilon$ can be found such that the update is successful and the $\mathrm{SQH}$ iteration proceeds until an appropriate stopping criterion is reached.

Our SQH scheme for differential Nash games is implemented as follows:

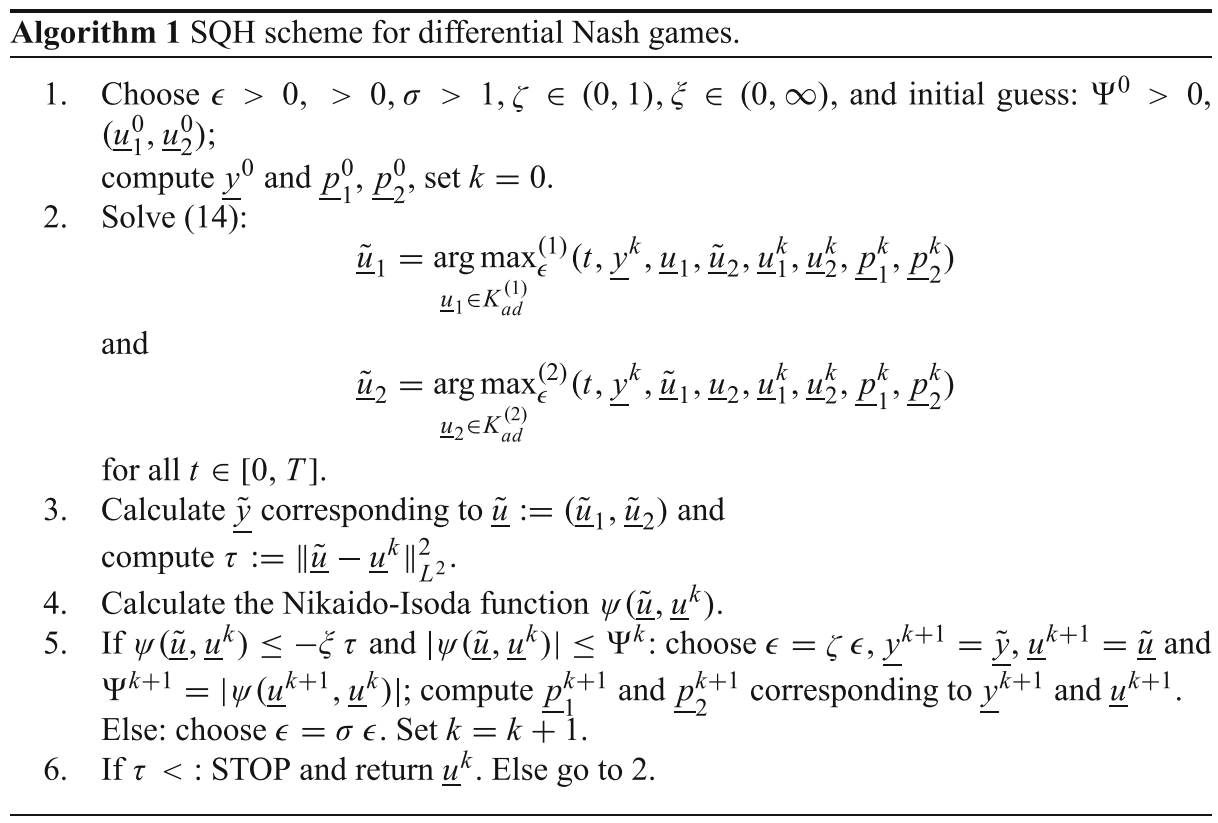

In the following proposition, we prove that the Steps 1-6 of the SQH scheme are well posed. For the proof, we consider the assumptions of Theorem 3.1 with further simplifying hypothesis, which can be relaxed at the cost of more involved calculations.

Our purpose is to show that it is possible to find an $\epsilon$ in Algorithm 1 such that $\underline{\tilde{u}}$ generated in Step 2 satisfies the criterion required in Step 5 for a successful update. We have

Proposition 3.2 Let the assumptions of Theorem 3.1 hold, and suppose that $f_{0}, g_{i}, \ell_{i}$, $i=1,2$ are twice continuous differentiable and strictly convex in $\underline{y}$ and $\underline{u}$ for all $t \in$ $[0, T]$. Moreover, let $M_{1}, M_{2}$ dependent only on $t$ and suppose that $\underline{f}_{0}, \ell_{i}$ and $g_{i}, i=1,2$, represent quadratic forms in $\underline{u}$ and $y$ such that their Hessians are constant.

Let $\left(\underline{\tilde{y}}, \underline{\tilde{u}}_{1}, \underline{\tilde{u}}_{2}\right),\left(\underline{y}^{k}, \underline{u}_{1}^{k}, \underline{u}_{2}^{k}\right)$ be generated by Algorithm 1, Steps $2-3$, and denote $\delta \underline{u}=$ $\underline{\tilde{u}}-\underline{u}^{k} . \bar{T}$ Then, there exists $a \theta>0$ independent of $\epsilon$ such that, for $\epsilon>0$ currently chosen in Step 2, the following inequality holds:

$$
\psi\left(\underline{\tilde{u}}, \underline{u}^{k}\right) \leq-(\epsilon-\theta)\|\delta \underline{u}\|_{L^{2}(0, T)}^{2} .
$$

In particular, if $\epsilon>\theta$ then $\psi\left(\underline{\tilde{u}}, \underline{u}^{k}\right) \leq 0$.

Proof Recall the definition of the Nikaido-Isoda function:

$$
\psi\left(\underline{\tilde{u}}, \underline{u}^{k}\right):=\tilde{J}_{1}\left(\underline{\tilde{u}}_{1}, \underline{\tilde{u}}_{2}\right)-\tilde{J}_{1}\left(\underline{u}_{1}^{k}, \underline{\tilde{u}}_{2}\right)+\tilde{J}_{2}\left(\underline{\tilde{u}}_{1}, \underline{\tilde{u}}_{2}\right)-\tilde{J}_{2}\left(\underline{\tilde{u}}_{1}, \underline{u}_{2}^{k}\right) .
$$

We focus on the first two terms involving $J_{1}$; however, the same calculation applies to the last two terms with $J_{2}$. 
Notice that, with our assumptions, the function $y\left(\underline{u}_{1}, \underline{u}_{2}\right)$ results differentiable. Denote $\underline{y}^{k}=\underline{y}\left(\underline{u}_{1}^{k}, \underline{u}_{2}^{k}\right), \underline{\tilde{y}}=\underline{y}\left(\underline{\tilde{u}}_{1}, \underline{\tilde{u}}_{2}\right)$ and $\underline{\tilde{y}}_{1}^{k}=\underline{y}\left(\underline{u}_{1}^{k}, \underline{\tilde{u}}_{2}\right), \underline{p}_{1}^{k}=\underline{p}_{1}\left(\underline{u}_{1}^{k}, \underline{u}_{2}^{k}\right)$, and define $\delta \underline{y}_{1}:=$ $\underline{\tilde{y}}-\underline{\tilde{y}}_{1}^{k}$, and $\delta \underline{\tilde{y}}_{1}:=\underline{\tilde{y}}_{1}^{k}-\underline{y}^{k}$.

Consider the augmented Hamiltonian $\mathcal{K}_{\epsilon}^{(1)}$ of player $P_{1}$. Similar computations can be done with $\mathcal{K}_{\epsilon}^{(2)}$. It holds

$$
\mathcal{K}_{\epsilon}^{(1)}\left(t, \underline{y}^{k}, \underline{\tilde{u}}_{1}, \underline{\tilde{u}}_{2}, \underline{u}_{1}^{k}, \underline{u}_{2}^{k}, \underline{p}_{1}^{k}, \underline{p}_{2}^{k}\right) \geq \mathcal{K}_{\epsilon}^{(1)}\left(t, \underline{y}^{k}, \underline{w}, \underline{\tilde{u}}_{2}, \underline{u}_{1}^{k}, \underline{u}_{2}^{k}, \underline{p}_{1}^{k}, \underline{p}_{2}^{k}\right),
$$

for any $\underline{w} \in K_{a d}^{(1)}$. Hence, choosing $\underline{w}=\underline{u}_{1}^{k}$, we have

$$
\begin{aligned}
\mathcal{K}_{\epsilon}^{(1)}\left(t, \underline{y}^{k}, \underline{\tilde{u}}_{1}, \underline{\tilde{u}}_{2}, \underline{u}_{1}^{k}, \underline{u}_{2}^{k}, \underline{p}_{1}^{k}, \underline{p}_{2}^{k}\right) & \geq \mathcal{K}_{\epsilon}^{(1)}\left(t, \underline{y}^{k}, \underline{u}_{1}^{k}, \underline{\tilde{u}}_{2}, \underline{u}_{1}^{k}, \underline{u}_{2}^{k}, \underline{p}_{1}^{k}, \underline{p}_{2}^{k}\right) \\
& =\mathcal{H}_{1}\left(t, \underline{y}^{k}, \underline{u}_{1}^{k}, \underline{\tilde{u}}_{2}, \underline{p}_{1}^{k}, \underline{p}_{2}^{k}\right)-\epsilon\left|\underline{\tilde{u}}_{2}-\underline{u}_{2}^{k}\right|^{2} .
\end{aligned}
$$

Now, compute

$$
\begin{aligned}
& J_{1}\left(\underline{\tilde{y}}_{\underline{\underline{u}}}, \underline{\tilde{u}}_{2}\right)-J_{1}\left(\underline{\tilde{y}}_{1}^{k}, \underline{u}_{1}^{k}, \underline{\tilde{u}}_{2}\right)= \\
& =\int_{0}^{T}\left(\ell_{1}\left(t, \underline{\tilde{y}}, \underline{\tilde{u}}_{1}, \underline{\tilde{u}}_{2}\right)-\ell_{1}\left(t, \underline{\tilde{y}}_{1}^{k}, \underline{u}_{1}^{k}, \underline{\tilde{u}}_{2}\right)\right) d t+g_{1}(\underline{\tilde{y}}(T))-g_{1}\left(\underline{\tilde{y}}_{1}^{k}(T)\right) \\
& +\int_{0}^{T}\left(\underline{p}_{1}^{k} \cdot \underline{f}\left(t, \underline{\tilde{y}}, \underline{\tilde{u}}_{1}, \underline{\tilde{u}}_{2}\right)-\underline{p}_{1}^{k} \cdot \underline{f}\left(t, \underline{\tilde{y}}, \underline{\tilde{u}}_{1}, \underline{\tilde{u}}_{2}\right)\right) d t \\
& +\int_{0}^{T}\left(\underline{p}_{1}^{k} \cdot \underline{f}\left(t, \underline{\tilde{y}}_{1}^{k}, \underline{u}_{1}^{k}, \underline{\tilde{u}}_{2}\right)-\underline{p}_{1}^{k} \cdot \underline{f}\left(t, \underline{\tilde{y}}_{1}^{k}, \underline{u}_{1}^{k}, \underline{\tilde{u}}_{2}\right)\right) d t \\
& =\int_{0}^{T}\left(-\mathcal{H}_{1}\left(t, \underline{\tilde{y}}, \underline{\tilde{u}}_{1}, \underline{\tilde{u}}_{2}, \underline{p}_{1}^{k}, \underline{p}_{2}^{k}\right)+\underline{p}_{1}^{k} \cdot \underline{f}\left(t, \underline{\tilde{y}}, \underline{\tilde{u}}_{1}, \underline{\tilde{u}}_{2}\right)\right) d t \\
& +\int_{0}^{T}\left(\mathcal{H}_{1}\left(t, \underline{\tilde{y}}_{1}^{k}, \underline{u}_{1}^{k}, \underline{\tilde{u}}_{2}, \underline{p}_{1}^{k}, \underline{p}_{2}^{k}\right)-\underline{p}_{1}^{k} \cdot \underline{f}\left(t, \underline{\tilde{y}}_{1}^{k}, \underline{u}_{1}^{k}, \underline{\tilde{u}}_{2}\right)\right) d t \\
& +g_{1}(\underline{\tilde{y}}(T))-g_{1}\left(\underline{\tilde{y}}_{1}^{k}(T)\right) \text {. }
\end{aligned}
$$

Notice that $\tilde{y}=\tilde{y}_{1}^{k}+\delta \underline{y}_{1}$. By applying the mean value theorem to second order to the maps $\left.\underline{y} \mapsto \mathcal{H}_{1} \overline{(\cdot}, \underline{y}, \cdot, \cdot \cdot, \cdot, \cdot\right)$ and $\underline{y} \mapsto \underline{f}(\cdot, \underline{y}, \cdot, \cdot)$, and $\underline{u}_{1} \mapsto \underline{f}\left(\cdot, \cdot, \underline{u}_{1}, \cdot\right)$, we have

$$
\begin{aligned}
& \mathcal{H}_{1}\left(t, \underline{\tilde{y}}_{1}^{k}+\delta \underline{y}_{1}, \underline{\tilde{u}}_{1}, \underline{\tilde{u}}_{2}, \underline{p}_{1}^{k}, \underline{p}_{2}^{k}\right)=\mathcal{H}_{1}\left(t, \underline{\tilde{y}}_{1}^{k}, \underline{\tilde{u}}_{1}, \underline{\tilde{u}}_{2}, \underline{p}_{1}^{k}, \underline{p}_{2}^{k}\right) \\
& +\left(\left(\underline{p}_{1}^{k}\right)^{\top} \partial_{y} \underline{f}_{0}\left(t, \underline{\tilde{y}}_{1}^{k}\right)-\partial_{y} \ell_{1}^{0}\left(t, \underline{\tilde{y}}_{1}^{k}\right)\right) \delta \underline{y}_{1}+\frac{1}{2} \delta \underline{y}_{1}^{\top} \partial_{y y}^{2} \mathcal{H}_{1} \delta \underline{y}_{1},
\end{aligned}
$$

and

$$
\underline{f}\left(t, \underline{\tilde{y}}_{1}^{k}+\delta \underline{y}_{1}, \underline{\tilde{u}}_{1}, \underline{\tilde{u}}_{2}\right)=\underline{f}\left(t, \underline{\tilde{y}}_{1}^{k}, \underline{\tilde{u}}_{1}, \underline{\tilde{u}}_{2}\right)+\partial_{y} \underline{f}_{0}\left(t, \underline{\tilde{y}}_{1}^{k}\right) \delta \underline{y}_{1}+\frac{1}{2} \delta \underline{y}_{1}^{\top} \partial_{y y}^{2} \underline{f}_{0} \delta \underline{y}_{1}
$$

and

$$
\underline{f}\left(t, \underline{\tilde{y}}_{1}^{k}, \underline{u}_{1}^{k}+\delta \underline{u}_{1}, \underline{\tilde{u}}_{2}\right)=\underline{f}\left(t, \underline{\tilde{y}}_{1}^{k}, \underline{u}_{1}^{k}, \underline{\tilde{u}}_{2}\right)+M_{1}(t) \delta \underline{u}_{1} .
$$


With these estimates, we continue the calculation above as follows. Notice that we add and subtract the term $\epsilon|\delta \underline{u}|^{2}$.

$$
\begin{aligned}
& J_{1}\left(\underline{\tilde{y}}, \underline{\tilde{u}}_{1}, \underline{\tilde{u}}_{2}\right)-J_{1}\left(\underline{\tilde{y}}_{1}^{k}, \underline{u}_{1}^{k}, \underline{\tilde{u}}_{2}\right)= \\
& \int_{0}^{T}\left(-\mathcal{H}_{1}\left(t, \underline{\tilde{y}}_{1}^{k}, \underline{\tilde{u}}_{1}, \underline{\tilde{u}}_{2}, \underline{p}_{1}^{k}, \underline{p}_{2}^{k}\right)+\mathcal{H}_{1}\left(t, \underline{\tilde{y}}_{1}^{k}, \underline{u}_{1}^{k}, \underline{\tilde{u}}_{2}, \underline{p}_{1}^{k}, \underline{p}_{2}^{k}\right)\right. \\
& \left.+\partial_{y} \ell_{1}^{0}\left(t, \underline{\tilde{y}}_{1}^{k}\right) \delta \underline{y}_{1}-\frac{1}{2} \delta \underline{y}_{1}^{\top} \partial_{y y}^{2} \mathcal{H}_{1} \delta \underline{y}_{1}+\left(\underline{p}_{1}^{k}\right)^{\top} M_{1}(t) \delta \underline{u}_{1}+\frac{1}{2}\left(\underline{p}_{1}^{k}\right)^{\top} \delta \underline{y}_{1}^{\top} \partial_{y y}^{2} \underline{f}_{0} \delta \underline{y}_{1}\right) d t \\
& +g_{1}\left(\underline{\tilde{y}}^{\mathrm{y}}(T)\right)-g_{1}\left(\underline{\tilde{y}}_{1}^{k}(T)\right)+\epsilon|\delta \underline{u}|^{2}-\epsilon \mid \delta \underline{u}^{2} \\
& =\int_{0}^{T}\left(-\mathcal{K}_{\epsilon}^{(1)}\left(t, \underline{\tilde{y}}_{1}^{k}, \underline{\tilde{u}}_{1}, \underline{\tilde{u}}_{2}, \underline{u}_{1}^{k}, \underline{u}_{2}^{k}, \underline{p}_{1}^{k}, \underline{p}_{2}^{k}\right)-\epsilon \mid \delta \underline{u}^{2}+\mathcal{H}_{1}\left(t, \underline{\tilde{y}}_{1}^{k}, \underline{u}_{1}^{k}, \underline{\tilde{u}}_{2}, \underline{p}_{1}^{k}, \underline{p}_{2}^{k}\right)\right. \\
& \left.+\partial_{y} \ell_{1}^{0}\left(t, \underline{\tilde{y}}_{1}^{k}\right) \delta \underline{y}_{1}-\frac{1}{2} \delta \underline{y}_{1}^{\top} \partial_{y y}^{2} \mathcal{H}_{1} \delta \underline{y}_{1}+\left(\underline{p}_{1}^{k}\right)^{\top} M_{1}(t) \delta \underline{u}_{1}+\frac{1}{2}\left(\underline{p}_{1}^{k}\right)^{\top} \delta \underline{y}_{1}^{\top} \partial_{y y}^{2} \underline{f}_{0} \delta \underline{y}_{1}\right) d t \\
& +\partial_{y} g_{1}\left(\underline{\tilde{y}}_{1}^{k}(T)\right) \delta \underline{y}_{1}(T)+\frac{1}{2} \delta \underline{y}_{1}(T)^{\top} \partial_{y y}^{2} g_{1} \delta \underline{y}_{1}(T) .
\end{aligned}
$$

Now, we consider the following integration by parts

$$
\begin{aligned}
& \int_{0}^{T}\left(\left(\underline{p}_{1}^{k}\right)^{\top} M_{1}(t) \delta \underline{u}_{1}+\frac{1}{2}\left(\underline{p}_{1}^{k}\right)^{\top} \delta \underline{y}_{1}^{\top} \partial_{y y}^{2} \underline{f}_{0} \delta \underline{y}_{1}\right) d t= \\
& -\partial_{y} g_{1}\left(\underline{\tilde{y}}_{1}^{k}(T)\right) \delta \underline{y}_{1}(T)-\int_{0}^{T}\left[\left(\underline{p}_{1}^{k}\right)^{\prime}+\left(\partial_{y} \underline{f}_{0}\left(t, \underline{\tilde{y}}_{1}^{k}\right)\right)^{\top} \underline{p}_{1}^{k}\right] \delta \underline{y}_{1} d t .
\end{aligned}
$$

Using this result in the previous calculation, we have

$$
\begin{aligned}
& \int_{0}^{T}\left(-\mathcal{K}_{\epsilon}^{(1)}\left(t, \underline{\tilde{y}}_{1}^{k}, \underline{\tilde{u}}_{1}, \underline{\tilde{u}}_{2}, \underline{u}_{1}^{k}, \underline{u}_{2}^{k}, \underline{p}_{1}^{k}, \underline{p}_{2}^{k}\right)-\epsilon|\delta \underline{u}|^{2}+\mathcal{H}_{1}\left(t, \underline{\tilde{y}}_{1}^{k}, \underline{u}_{1}^{k}, \underline{\tilde{u}}_{2}, \underline{p}_{1}^{k}, \underline{p}_{2}^{k}\right)\right. \\
& \left.-\frac{1}{2} \delta \underline{y}_{1}^{\top} \partial_{y y}^{2} \mathcal{H}_{1} \delta \underline{y}_{1}\right) d t+\frac{1}{2} \delta \underline{y}_{1}(T)^{\top} \partial_{y y}^{2} g_{1} \delta \underline{y}_{1}(T) \\
& +\int_{0}^{T}\left[-\left(\underline{p}_{1}^{k}\right)^{\prime}-\left(\partial_{y} \underline{f}_{0}\left(t, \underline{\tilde{y}}_{1}^{k}\right)\right)^{\top} \underline{p}_{1}^{k}+\partial_{y} \ell_{1}^{0}\left(t, \underline{\tilde{y}}_{1}^{k}\right)\right] \delta \underline{y}_{1} d t .
\end{aligned}
$$

Applying again the mean value theorem to second order to the maps $\underline{y} \mapsto \mathcal{H}_{1}(\cdot, \underline{y}, \cdot, \cdot, \cdot, \cdot)$ and $\underline{y} \mapsto \mathcal{K}_{\epsilon}^{(1)}(\cdot, \underline{y}, \cdot, \cdot, \cdot, \cdot, \cdot, \cdot)$, we get

$$
\begin{aligned}
& \mathcal{H}_{1}\left(t, \underline{y}^{k}+\delta \underline{\tilde{y}}_{1}, \underline{u}_{1}^{k}, \underline{\tilde{u}}_{2}, \underline{p}_{1}^{k}, \underline{p}_{2}^{k}\right)=\mathcal{H}_{1}\left(t, \underline{y}^{k}, \underline{u}_{1}^{k}, \underline{\tilde{u}}_{2}, \underline{p}_{1}^{k}, \underline{p}_{2}^{k}\right) \\
& +\left(\left(\underline{p}_{1}^{k}\right)^{\top} \partial_{y} \underline{f}_{0}\left(t, \underline{y}^{k}\right)-\partial_{y} \ell_{1}^{0}\left(t, \underline{y}^{k}\right)\right) \delta \underline{\tilde{y}}_{1}+\frac{1}{2} \delta \underline{\tilde{y}}_{1}^{\top} \partial_{y y}^{2} \mathcal{H}_{1} \delta \underline{\tilde{y}}_{1},
\end{aligned}
$$

and

$$
\begin{aligned}
& \mathcal{K}_{\epsilon}^{(1)}\left(t, \underline{y}^{k}+\delta \underline{\tilde{y}}_{1}, \underline{\tilde{u}}_{1}, \underline{\tilde{u}}_{2}, \underline{u}_{1}^{k}, \underline{u}_{2}^{k}, \underline{p}_{1}^{k}, \underline{p}_{2}^{k}\right)=\mathcal{K}_{\epsilon}^{(1)}\left(t, \underline{y}^{k}, \underline{\tilde{u}}_{1}, \underline{\tilde{u}}_{2}, \underline{u}_{1}^{k}, \underline{u}_{2}^{k}, \underline{p}_{1}^{k}, \underline{p}_{2}^{k}\right) \\
& +\left(\left(\underline{p}_{1}^{k}\right)^{\top} \partial_{y} \underline{f}_{0}\left(t, \underline{y}^{k}\right)-\partial_{y} \ell_{1}^{0}\left(t, \underline{y}^{k}\right)\right) \delta \underline{\tilde{y}}_{1}+\frac{1}{2} \delta \underline{\tilde{y}}_{1}^{\top} \partial_{y y}^{2} \mathcal{H}_{1} \delta \underline{\tilde{y}}_{1},
\end{aligned}
$$


With these results and replacing the expression of $-\left(\underline{p}_{1}^{k}\right)^{\prime}$, we continue the calculation as follows:

$$
\begin{aligned}
& J_{1}\left(\underline{\tilde{y}}_{\underline{\underline{u}}}, \underline{\tilde{u}}_{2}\right)-J_{1}\left(\underline{\tilde{y}}_{1}^{k}, \underline{u}_{1}^{k}, \underline{\tilde{u}}_{2}\right)= \\
& \int_{0}^{T}\left(-\mathcal{K}_{\epsilon}^{(1)}\left(t, \underline{y}^{k}, \underline{\tilde{u}}_{1}, \underline{\tilde{u}}_{2}, \underline{u}_{1}^{k}, \underline{u}_{2}^{k}, \underline{p}_{1}^{k}, \underline{p}_{2}^{k}\right)+\mathcal{H}_{1}\left(t, \underline{y}^{k}, \underline{u}_{1}^{k}, \underline{\tilde{u}}_{2}, \underline{p}_{1}^{k}, \underline{p}_{2}^{k}\right)-\epsilon \mid \delta \underline{u}^{2}\right. \\
& \left.-\frac{1}{2} \delta \underline{y}_{1}^{\top} \partial_{y y}^{2} \mathcal{H}_{1} \delta \underline{y}_{1}\right) d t+\frac{1}{2} \delta \underline{y}_{1}(T)^{\top} \partial_{y y}^{2} g_{1} \delta \underline{y}_{1}(T) \\
& +\int_{0}^{T}\left(\left(\underline{p}_{1}^{k}\right)^{\top}\left[\partial_{y} \underline{f}_{0}\left(t, \underline{y}^{k}\right)-\partial_{y} \underline{f}_{0}\left(t, \underline{\tilde{y}}_{1}^{k}\right)\right]+\partial_{y} \ell_{1}^{0}\left(t, \underline{\tilde{y}}_{1}^{k}\right)-\partial_{y} \ell_{1}^{0}\left(t, \underline{y}^{k}\right)\right) \delta \underline{y}_{1} d t \\
& \leq \int_{0}^{T}\left(-\epsilon\left|\delta \underline{u}_{1}\right|^{2}-\frac{1}{2} \delta \underline{y}_{1}^{\top} \partial_{y y}^{2} \mathcal{H}_{1} \delta \underline{y}_{1}\right) d t+\frac{1}{2} \delta \underline{y}_{1}(T)^{\top} \partial_{y y}^{2} g_{1} \delta \underline{y}_{1}(T) \\
& +\int_{0}^{T}\left(\delta \underline{y}_{1}^{\top} \partial_{y y}^{2} \ell_{1}^{0} \delta \underline{\tilde{y}}_{1}-\left(\underline{p}_{1}^{k}\right)^{\top} \delta \underline{y}_{1}^{\top} \partial_{y y}^{2} \underline{f}_{0} \delta \underline{\tilde{y}}_{1}\right) d t,
\end{aligned}
$$

where in the last integral we applied the mean value theorem to the maps $\underline{y} \mapsto \partial_{y} \ell_{1}^{0}(\cdot, \underline{y})$ and $\underline{y} \mapsto \partial_{y} \underline{f}_{0}(\cdot, \underline{y})$.

Therefore, we have shown that

$$
\begin{aligned}
J_{1}\left(\underline{\tilde{y}}, \underline{\tilde{u}}_{1}, \underline{\tilde{u}}_{2}\right)-J_{1}\left(\underline{\tilde{y}}_{1}^{k}, \underline{u}_{1}^{k}, \underline{\tilde{u}}_{2}\right) \leq & \int_{0}^{T}\left(-\epsilon\left|\delta \underline{u}_{1}\right|^{2}-\frac{1}{2} \delta \underline{y}_{1}^{\top} \partial_{y y}^{2} \mathcal{H}_{1} \delta \underline{y}_{1}\right. \\
& \left.+\delta \underline{y}_{1}^{\top} \partial_{y y}^{2} \ell_{1}^{0} \delta \underline{\tilde{y}}_{1}-\left(\underline{p}_{1}^{k}\right)^{\top} \delta \underline{y}_{1}^{\top} \partial_{y y}^{2} \underline{f}_{0} \delta \underline{\tilde{y}}_{1}\right) d t \\
& +\frac{1}{2} \delta \underline{y}_{1}(T)^{\top} \partial_{y y}^{2} g_{1} \delta \underline{y}_{1}(T) .
\end{aligned}
$$

With a similar computation, we also obtain

$$
\begin{aligned}
J_{2}\left(\underline{\tilde{y}}, \underline{\tilde{u}}_{1}, \underline{\tilde{u}}_{2}\right)-J_{2}\left(\underline{\tilde{y}}_{2}^{k}, \underline{\tilde{u}}_{1}, \underline{u}_{2}^{k}\right) \leq & \int_{0}^{T}\left(-\epsilon\left|\delta \underline{u}_{2}\right|^{2}-\frac{1}{2} \delta \underline{y}_{2}^{\top} \partial_{y y}^{2} \mathcal{H}_{2} \delta \underline{y}_{2}\right. \\
& \left.+\delta \underline{y}_{2}^{\top} \partial_{y y}^{2} \ell_{2}^{0} \delta \underline{\tilde{y}}_{2}-\left(\underline{p}_{2}^{k}\right)^{\top} \delta \underline{y}_{2}^{\top} \partial_{y y}^{2} \underline{f}_{0} \delta \underline{\tilde{y}}_{2}\right) d t \\
& +\frac{1}{2} \delta \underline{y}_{2}(T)^{\top} \partial_{y y}^{2} g_{2} \delta \underline{y}_{2}(T),
\end{aligned}
$$

where $\underline{\tilde{y}}_{2}^{k}=\underline{y}\left(\underline{\tilde{u}}_{1}, \underline{u}_{2}^{k}\right), \delta \underline{y}_{2}:=\underline{\tilde{y}}-\underline{\tilde{y}}_{2}^{k}$ and $\delta \underline{\tilde{y}}_{2}:=\underline{\tilde{y}}_{2}^{k}-\underline{y}^{k}$. Thus, we arrive at the following inequality:

$$
\begin{aligned}
\psi\left(\underline{\tilde{u}}, \underline{u}^{k}\right) \leq & \int_{0}^{T}\left(-\epsilon\left[\left|\delta \underline{u}_{1}\right|^{2}+\left|\delta \underline{u}_{2}\right|^{2}\right]-\frac{1}{2} \delta \underline{y}_{1}^{\top} \partial_{y y}^{2} \mathcal{H}_{1} \delta \underline{y}_{1}-\frac{1}{2} \delta \underline{y}_{2}^{\top} \partial_{y y}^{2} \mathcal{H}_{2} \delta \underline{y}_{2}\right. \\
& \left.+\delta \underline{y}_{1}^{\top} \partial_{y y}^{2} \ell_{1}^{0} \delta \underline{\tilde{y}}_{1}-\left(\underline{p}_{1}^{k}\right)^{\top} \delta \underline{y}_{1}^{\top} \partial_{y y}^{2} \underline{f}_{0} \delta \underline{\tilde{y}}_{1}+\delta \underline{y}_{2}^{\top} \partial_{y y}^{2} \ell_{2}^{0} \delta \underline{\tilde{y}}_{2}-\left(\underline{p}_{2}^{k}\right)^{\top} \delta \underline{y}_{2}^{\top} \partial_{y y}^{2} \underline{f}_{0} \delta \underline{\tilde{y}}_{2}\right) d t \\
& +\frac{1}{2} \delta \underline{y}_{1}(T)^{\top} \partial_{y y}^{2} g_{1} \delta \underline{y}_{1}(T)+\frac{1}{2} \delta \underline{y}_{2}(T)^{\top} \partial_{y y}^{2} g_{2} \delta \underline{y}_{2}(T) .
\end{aligned}
$$

Next, we notice that the solutions to the state and adjoint problems are uniformly bounded in $[0, T]$ for any choice of $\underline{u} \in U_{a d}$, and the following estimates hold:

$$
\left|\delta \underline{y}_{1}(t)\right| \leq \mathcal{C}_{11}\left\|\delta \underline{u}_{1}\right\|_{L^{2}(0, T)}, \quad\left|\delta \underline{y}_{2}(t)\right| \leq \mathcal{C}_{22}\left\|\delta \underline{u}_{2}\right\|_{L^{2}(0, T)}, \quad t \in(0, T) .
$$


Similarly,

$$
\left|\delta \underline{\tilde{y}}_{1}(t)\right| \leq \mathcal{C}_{12}\left\|\delta \underline{u}_{2}\right\|_{L^{2}(0, T)}, \quad\left|\delta \underline{\tilde{y}}_{2}(t)\right| \leq \mathcal{C}_{21}\left\|\delta \underline{u}_{1}\right\|_{L^{2}(0, T)}, \quad t \in(0, T) ;
$$

see [9] for a proof. By using these estimates in Eq. 19, we obtain

$$
\psi\left(\underline{\tilde{u}}, \underline{u}^{k}\right) \leq-(\epsilon-\theta)\|\delta \underline{u}\|_{L^{2}(0, T)}^{2},
$$

where $\theta$ depends on the functions computed at the $k$ th iteration but not on $\epsilon$. Thus, the claim is proved.

We remark that in Step 2 of the SQH algorithm, the NE solution $\underline{\tilde{u}}$ obtained in Step 2 depends on $\epsilon$ so that $\left\|\underline{\tilde{u}}-\underline{u}^{k}\right\|_{L^{2}(0, T)}^{2}$ decreases as $O\left(1 / \epsilon^{2}\right)$. In order to illustrate this fact, consider the following optimisation problem:

$$
\max f_{\epsilon}(u):=b u-\frac{v}{2} u^{2}-\epsilon(u-v)^{2},
$$

where $v, \epsilon>0$. Clearly, the function $f_{\epsilon}$ is concave and its maximum is attained at $\tilde{u}=$ $(b+2 \epsilon v) /(v+2 \epsilon)$. Furthermore, we have

$$
|\tilde{u}-v|=\frac{|b-v v|}{(v+2 \epsilon)} .
$$

Now, subject to the assumptions of Proposition 3.2 and using the estimates in its proof, we can state that there exists a constant $C>0$ such that

$$
\left|\psi\left(\underline{\tilde{u}}, \underline{u}^{k}\right)\right| \leq C\left\|\underline{\tilde{u}}-\underline{u}^{k}\right\|_{L^{2}(0, T)}^{2},
$$

where $C$ increases linearly with $\epsilon$. On the other hand, since the HP functions are concave, we have that $\left\|\underline{\tilde{u}}-\underline{u}^{k}\right\|_{L^{2}(0, T)}^{2}$ decreases as $O\left(1 / \epsilon^{2}\right)$. Therefore, given the value $\Psi^{k}$ in Step 5 of the SQH algorithm, it is always possible to choose $\epsilon$ sufficiently large such that $\left|\psi\left(\underline{\tilde{u}}, \underline{u}^{k}\right)\right| \leq$ $\Psi^{k}$.

In Algorithm 1, we have that $\psi\left(\underline{u}^{k+1}, \underline{u}^{k}\right) \rightarrow 0$ as $k \rightarrow \infty$. Thus, since $\psi\left(\underline{u}^{k+1}, \underline{u}^{k}\right) \leq$ $-\xi\left\|\underline{u}^{k+1}-\underline{u}^{k}\right\|_{L^{2}}^{2}$, it follows that $\lim _{k}\left\|\underline{u}^{k+1}-\underline{u}^{k}\right\|_{L^{2}}^{2}=0$ and hence the convergence criterion in Step 6 can be satisfied.

We remark that, subject to the assumptions of Proposition 3.2, if $\left(\underline{u}_{1}^{k}, \underline{u}_{2}^{k}\right)$ generated by Algorithm 1 satisfies the PMP conditions, then Algorithm 1 stops returning $\left(\underline{u}_{1}^{k}, \underline{u}_{2}^{k}\right)$.

\section{Numerical Experiments}

In this section, we present results of four numerical experiments to validate the computational performance of the proposed SQH scheme. We remark that in all these experiments the structure of the corresponding problems is such that in Step 2 of the SQH algorithm the update $\underline{\tilde{u}}(t)$ at any fixed $t$ can be determined analytically.

The first experiment exploits the possibility to compute open-loop NE solutions to linearquadratic Nash games by solving a coupled system of Riccati equations [14]. Thus, we use this solution for comparison to the solution of the same Nash game obtained with the SQH method.

Our linear-quadratic Nash game is formulated as follows:

$$
\underline{y}^{\prime}(t)=A \underline{y}(t)+B_{1} \underline{u}_{1}(t)+B_{2} \underline{u}_{2}(t), \quad \underline{y}(0)=\underline{y}_{0},
$$


where

$$
A=\left(\begin{array}{ll}
1 & 0 \\
0 & 2
\end{array}\right), \quad B_{1}=\left(\begin{array}{cc}
1 & 0 \\
0 & -1
\end{array}\right), \quad B_{2}=\left(\begin{array}{cc}
2 & -1 \\
0 & 2
\end{array}\right), \quad \underline{y}_{0}=\left(\begin{array}{l}
2 \\
1
\end{array}\right)
$$

Therefore, $y(t) \in \mathbb{R}^{2}$ and $\underline{u}_{i}(t) \in \mathbb{R}^{2}, t \in[0, T]$.

The cost functionals are as follows:

$$
J_{i}\left(\underline{y}, \underline{u}_{1}, \underline{u}_{2}\right)=\frac{1}{2} \int_{0}^{T}\left(\underline{y}(s)^{\top} L_{i}(s) \underline{y}(s)+\underline{u}_{i}(s)^{\top} N_{i}(s) \underline{u}_{i}(s)\right) d s+\frac{1}{2} \underline{y}(T)^{\top} D_{i} \underline{y}(T),
$$

$i=1,2$, where the matrices $L_{i}(s), D_{i}, N_{i}(s)$ are given by $L_{1}=\alpha_{1} I_{2}, L_{2}=\alpha_{2} I_{2}$, $N_{1}=v_{1} I_{2}, N_{2}=v_{2} I_{2}$, and $D_{1}=\gamma_{1} I_{2}$, and $D_{2}=\gamma_{2} I_{2}$, where $I_{2}$ is the identity matrix in $\mathbb{R}^{2}$. In the following experiment, we choose $\alpha_{1}=1, \alpha_{2}=10, v_{1}=0.1, v_{2}=0.1, \gamma_{1}=0$ and $\gamma_{2}=0$.

We consider the time interval $[0, T]$, with $T=0.2$, subdivided into $N=2000$ subintervals and, on this grid, the state and adjoint equations are solved numerically by a midpoint scheme [3].

The initial guess $\underline{u}^{0}$ for the SQH iteration are zero functions, and we choose $\epsilon=10$, $\zeta=0.95, \sigma=1.05, \xi=10^{-8}, \Psi^{0}=10$, and $\mathcal{K}=10^{-14}$. With this setting, we obtain the Nash strategies $\left(\underline{u}_{1}, \underline{u}_{2}\right)$ depicted in Fig. 1 (left), which are compared with the solution
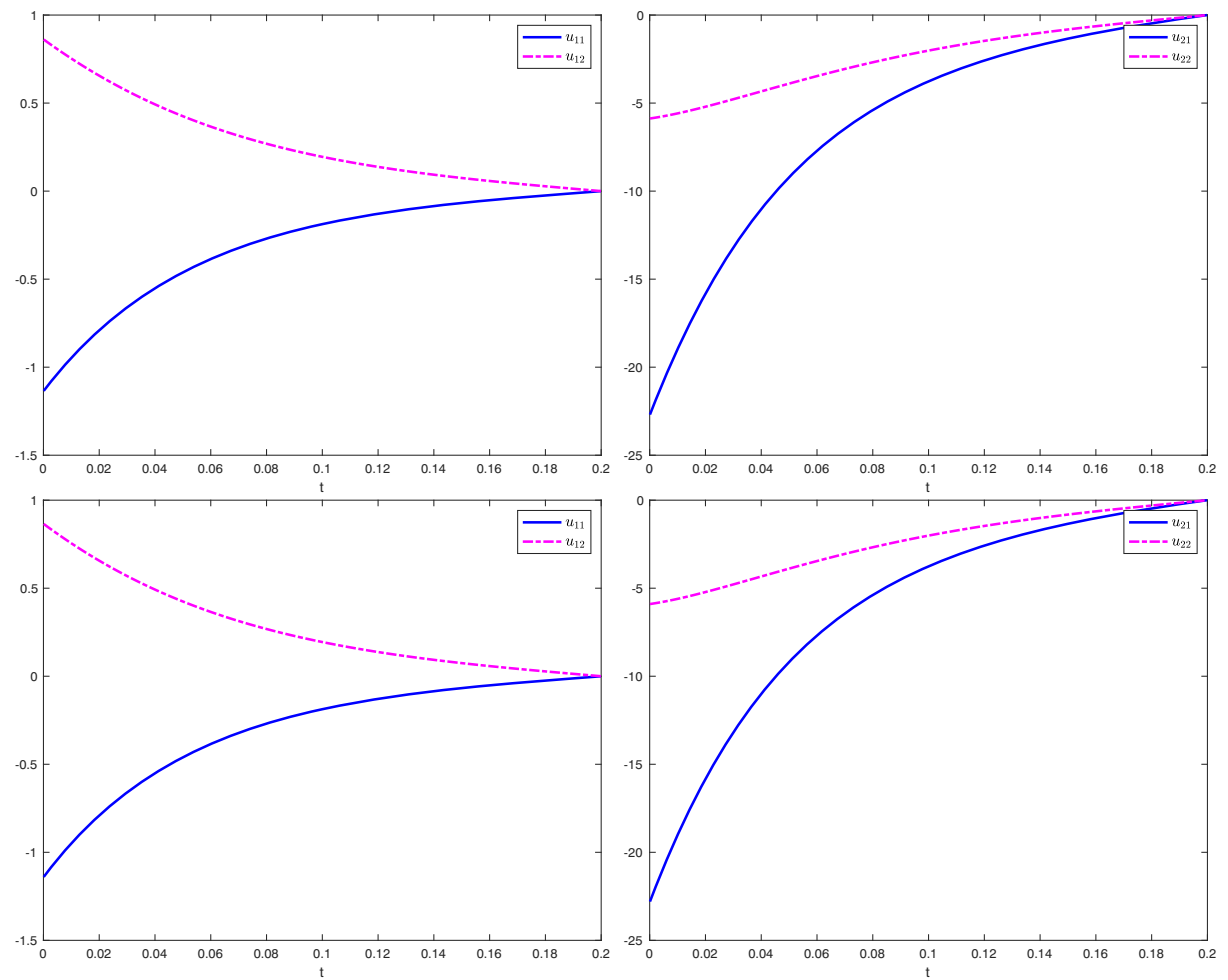

Fig. 1 Strategies $\underline{u}_{1}$ (left) and $\underline{u}_{2}$ for the LQ Nash game obtained with the SQH scheme (top) and by solving the Riccati system (bottom) 

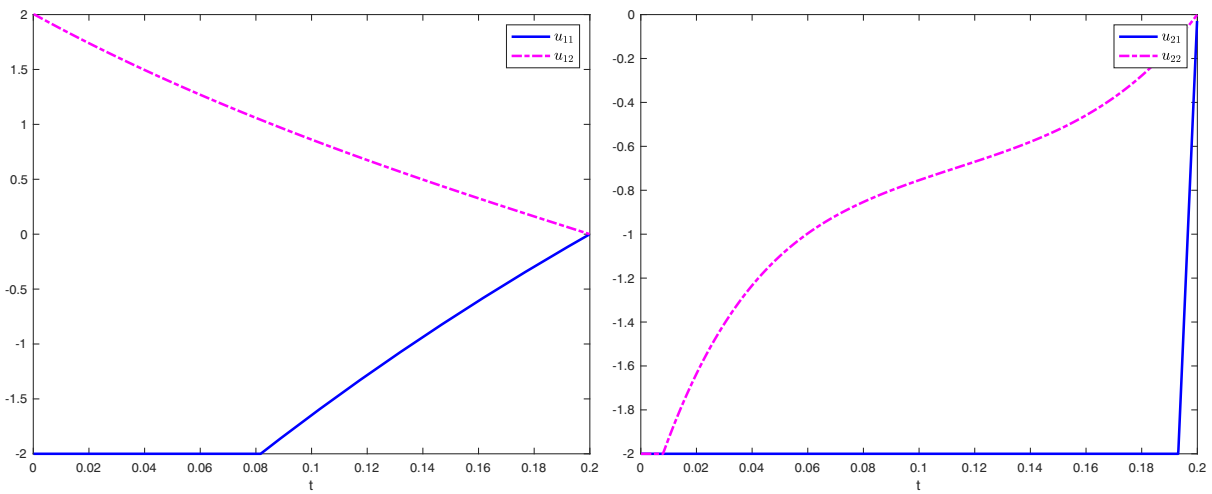

Fig. 2 Strategies $\underline{u}_{1}$ (left) and $\underline{u}_{2}$ for the LQ Nash game with constraints on $\underline{u}$ as obtained by the SQH scheme

obtained by solving the Riccati system as shown in Fig. 1 (right). We can see that the two sets of solutions overlap.

Next, we consider the same setting but require that the players' strategies are constrained by choosing $K_{a d}^{(i)}=[-2,2] \times[-2,2], i=1,2$. With this setting, we obtain the strategies depicted in Fig. 2.

In our third experiment, we consider a setting similar to the second experiment but add to the cost functionals a weighted $L^{1}$ cost of the strategies. We have (written in a more compact form):

$$
J_{i}\left(\underline{y}, \underline{u}_{1}, \underline{u}_{2}\right)=\frac{1}{2} \int_{0}^{T}\left(\alpha_{i}|\underline{y}(s)|^{2}+v_{i}\left|\underline{u}_{i}(s)\right|^{2}+2 \beta_{i}\left|\underline{u}_{i}(s)\right|\right) d s,
$$

where $i=1,2$; the terms with $D_{i}, i=1,2$, are omitted. We choose $v_{1}=0.1, v_{2}=$ 0.01 , and $\beta_{1}=0.01, \beta_{2}=0.1$; the other parameters are set as in the first experiment. Furthermore, we require that the players' strategies are constrained by choosing $K_{a d}^{(i)}=$ $[-10,10] \times[-10,10], i=1,2$. The strategies obtained with this setting are depicted in Fig. 3. Notice that the addition of $L^{1}$ costs of the players' actions promotes their sparsity.
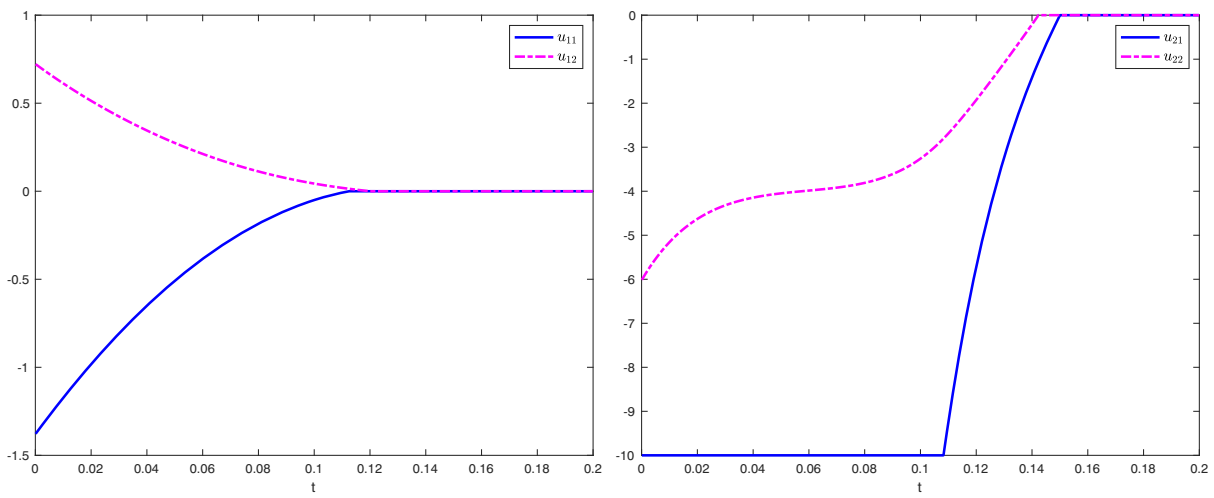

Fig. 3 Strategies $\underline{u}_{1}$ (left) and $\underline{u}_{2}$ for the Nash game with $L^{2}$ and $L^{1}$ costs and constraints on $\underline{u}$ as obtained by the SQH scheme 
In the next experiment, we consider a tracking problem where the cost functionals have the following structure:

$$
\begin{aligned}
J_{i}\left(\underline{y}, \underline{u}_{1}, \underline{u}_{2}\right)= & \frac{1}{2} \int_{0}^{T}\left(\alpha_{i}\left|\underline{y}(s)-\underline{y}^{i}(s)\right|^{2}+v_{i}\left|\underline{u}_{i}(s)\right|^{2}+2 \beta_{i}\left|\underline{u}_{i}(s)\right|\right) d s \\
& +\frac{\gamma_{i}}{2}\left|\underline{y}(T)-\underline{y}^{i}(T)\right|^{2},
\end{aligned}
$$
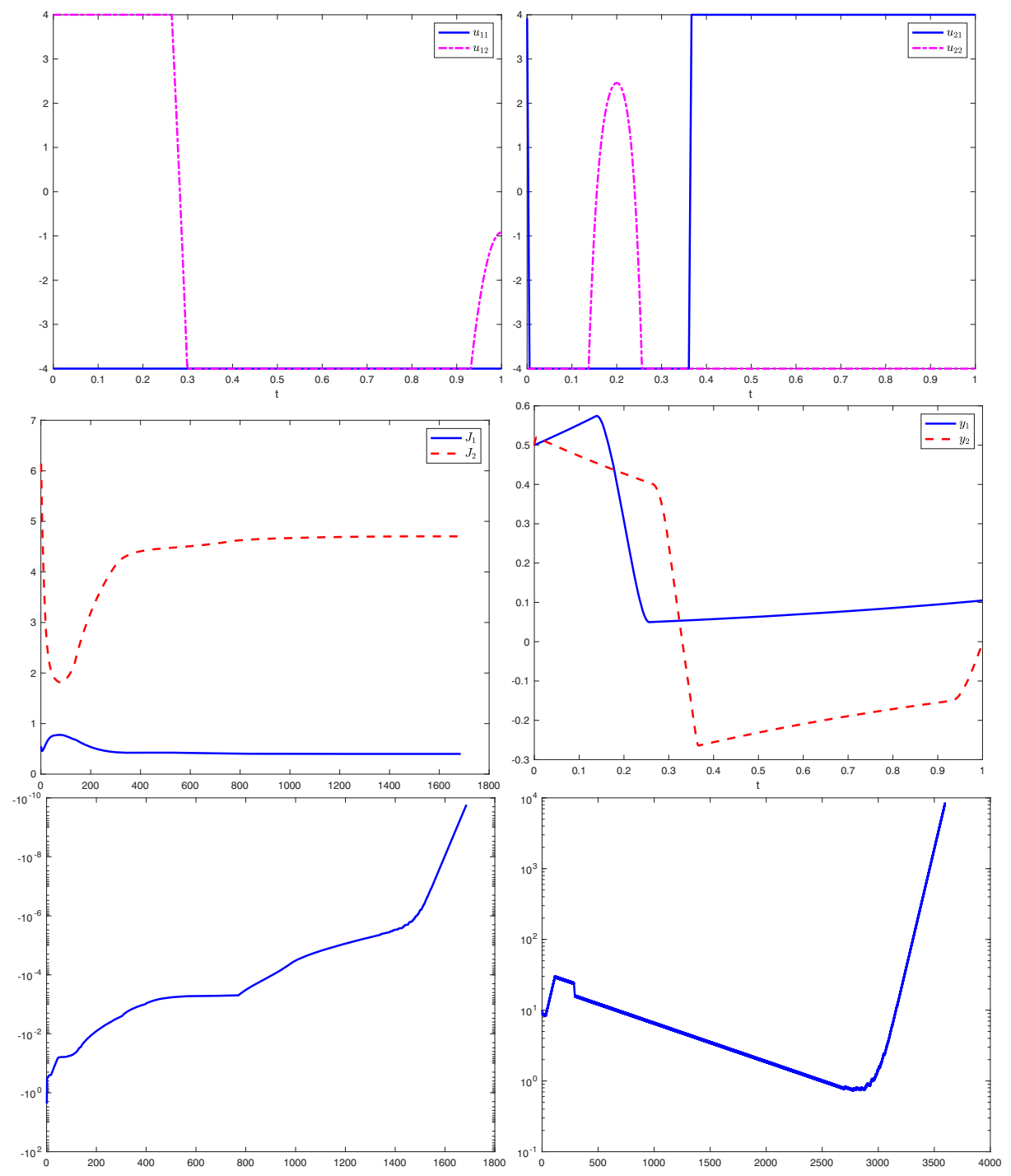

Fig. 4 Strategies $u_{1}$ (top, left) and $u_{2}$ (top, right) for the Nash game with tracking functional as obtained by the SQH scheme. In the middle figures, the values of $J_{1}$ and $J_{2}$ along the SQH iterations (left) and the evolution of $y$ corresponding to $\underline{u}_{1}$ and $\underline{u}_{2}$ (right). In the bottom figures, the values of $\psi$ (left) and of $\epsilon$ along the SQH iterāions 
where $\underline{y}^{i}$ denotes the trajectory desired by the Player $P_{i}, i=1,2$. Specifically, we take

$$
\underline{\bar{y}}^{1}(t)=\left(\begin{array}{l}
1 \\
1
\end{array}\right) \sin (2 \pi t), \quad \underline{y}^{2}(t)=\left(\begin{array}{l}
1 \\
1
\end{array}\right) \cos (2 \pi t) .
$$

Notice that these trajectories are orthogonal to each other, that is, the two players have very different purposes. For the initial state, we take $\underline{y}_{0}=(1 / 2,1 / 2)$.

In this fourth experiment, the values of the game parameters are given by $\alpha_{1}=1, \alpha_{2}=$ $10, v_{1}=10^{-8}, v_{2}=10^{-6}, \beta_{1}=10^{-8}, \beta_{2}=10^{-6}$, and $\gamma_{1}=1$ and $\gamma_{2}=1$. Furthermore, we require that the players' strategies are constrained by choosing $K_{a d}^{(i)}=[-4,4] \times[-4,4]$, $i=1,2$. In this experiment, we take $T=1$ and $N=10^{4}$ subdivision of $[0, T]$ for the numerical approximation. The parameters of the SQH scheme remain unchanged. The results of this experiment are depicted in Fig. 4.

For this concluding experiment, we report that the convergence criterion is achieved after $3593 \mathrm{SQH}$ iterations, whereas the number of successful updates is 1686 . Correspondingly, we see that $\psi$ is always negative and its absolute value monotonically decreases, with $\psi=$ $-1.70 \times 10^{-10}$ at convergence. On the other hand, we can see that the value of $\epsilon$ is changed along the iteration, while the values of the players' functionals reach the Nash equilibrium. The CPU time for this experiment is $1151.4 \mathrm{~s}$ on a laptop computer.

\section{Conclusion}

A sequential quadratic Hamiltonian (SQH) scheme for solving open-loop differential Nash games was discussed. Theoretical and numerical results were presented that successfully demonstrated the well-posedness and computational performance of the SQH method applied to different Nash games governed by ordinary differential equations.

However, the applicability of the proposed method seems not restricted to these models and appears to be a promising technique for solving infinite-dimensional differential Nash game problems that have been considered recently in different fields of applied mathematics; see, e.g., [10, 28, 31].

Acknowledgements We are very grateful to Abderrahmane Habbal and Souvik Roy for many insightful discussions on the topic of differential games. We especially thank Andrei Fursikov for continued support of our work.

Funding Open Access funding enabled and organized by Projekt DEAL.

Open Access This article is licensed under a Creative Commons Attribution 4.0 International License, which permits use, sharing, adaptation, distribution and reproduction in any medium or format, as long as you give appropriate credit to the original author(s) and the source, provide a link to the Creative Commons licence, and indicate if changes were made. The images or other third party material in this article are included in the article's Creative Commons licence, unless indicated otherwise in a credit line to the material. If material is not included in the article's Creative Commons licence and your intended use is not permitted by statutory regulation or exceeds the permitted use, you will need to obtain permission directly from the copyright holder. To view a copy of this licence, visit http://creativecommons.org/licenses/by/4.0/. 


\section{References}

1. Albul A, Sokolov B, Chernous'ko F. A method of calculating the control in antagonistic situations. USSR Comput Math Math Phys. 1978;18(5):21-27.

2. Boltyanskiı̌ VG, Gamkrelidze RV, Pontryagin LS. On the theory of optimal processes. Dokl Akad Nauk SSSR (NS). 1956;110:7-10.

3. Borzì A. Modelling with ordinary differential equations: a comprehensive approach. Numerical analysis and scientific computing series. Abingdon and Boca Raton: Chapman \& Hall/CRC; 2020.

4. Breitenbach T, Borzì A. On the SQH scheme to solve nonsmooth PDE optimal control problems. Numer Funct Anal Optim. 2019;40:1489-1531.

5. Breitenbach T, Borzì A. A sequential quadratic Hamiltonian method for solving parabolic optimal control problems with discontinuous cost functionals. J Dyn Control Syst. 2019;25:403-435.

6. Breitenbach T, Borzì A. The Pontryagin maximum principle for solving Fokker-Planck optimal control problems. Computational Optimization and Applied Mathematics. 2020;76:499-533.

7. Breitenbach T, Borzì A. A sequential quadratic Hamiltonian scheme for solving non-smooth quantum control problems with sparsity. J Comput Appl Math. 2020;369:112583.

8. Bressan A. Noncooperative differential games. Milan J Math. 2011;79(2):357-427.

9. Calà Campana F, Ciaramella G, Borzì A. Nash equilibria and bargaining solutions of differential bilinear games. Dynamic Games and Applications. 2020.

10. Chamekh R, Habbal A, Kallel M, Zemzemi N. A Nash game algorithm for the solution of coupled conductivity identification and data completion in cardiac electrophysiology. Mathematical Modelling of Natural Phenomena. 2019;14(2):15.

11. Chernous'ko FL, Lyubushin AA. Method of successive approximations for solution of optimal control problems. Optimal Control Applications and Methods. 1982;3(2):101-114.

12. Dmitruk AV, Osmolovskii NP. On the proof of Pontryagin's maximum principle by means of needle variations. J Math Sci. 2016;218(5):581-598.

13. Dockner E, Jørgensen S, Van Long N, Sorger G. Differential games in economics and management science. Cambridge: Cambridge University Press; 2000.

14. Engwerda J. LQ dynamic optimization and differential games. Chichester: Wiley; 2005.

15. Friedman A. Differential games. Wiley-Interscience. 1971.

16. Isaacs R. Differential games: a mathematical theory with applications to warfare and pursuit, control and optimization. New York: Wiley; 1965.

17. Järmark B. A new convergence control technique in differential dynamic programming. Technical Report TRITA-REG-7502, The Royal Institute of Technology, Stockholm, Sweden, Department of Automatic Control. 1975.

18. Jørgensen S, Zaccour G. Differential games in marketing. Springer US. 2003.

19. Kelley HJ, Kopp RE, Moyer HG. Successive approximation techniques for trajectory optimization. In: Proc. IAS symp. on vehicle system optimization; 1961. p. 10-25. New York.

20. Krylov IA, Chernous'ko FL. On a method of successive approximations for the solution of problems of optimal control. USSR Comput Math Math Phys. 1963;2(6):1371-1382. Transl of Zh Vychisl Mat Mat Fiz. 1962;2(6):1132-1139.

21. Krylov IA, Chernous'ko FL. An algorithm for the method of successive approximations in optimal control problems. USSR Comput Math Math Phys. 1972;12(1):15-38.

22. Nash JF. Equilibrium points in n-person games. Proceedings of the National Academy of Sciences. 1950;36(1):48-49.

23. Nash JF. Non-cooperative games. Ann Math. 1951;54(2):286-295.

24. Nikaido $\mathrm{H}$, Isoda $\mathrm{K}$. Note on noncooperative convex games. Pacific Journal of Mathematics. 1955;5:807-815.

25. Pontryagin LS. On the theory of differential games. Russian Mathematical Surveys. 1966;21(4):193246. Uspekhi Mat Nauk. 1966;21(4, 130):219-274.

26. Pontryagin LS. Linear differential games. SIAM Journal on Control. 1974;12(2):262-267.

27. Pontryagin LS, Boltyanskiǐ VG, Gamkrelidze RV, Mishchenko EF. The mathematical theory of optimal processes. New York-London: Wiley; 1962.

28. Roca Leon E, Le Pape A, Costes M, Desideri J-A, Alfano D. Concurrent aerodynamic optimization of rotor blades using a Nash game method. Journal of the American Helicopter Society. 2016;61(2):13.

29. Rockafellar RT. Monotone operators and the proximal point algorithm. SIAM J Control Optim. 1976;14(5):877-898.

30. Rockafellar RT, Wets RJ-B, Vol. 317. Variational analysis. Berlin Heidelberg: Springer-Verlag; 2009. 
31. Roy S, Borzì A, Habbal A. Pedestrian motion modelled by Fokker-Planck Nash games. Royal Society Open Science. 2017;4:170648.

32. Rozonoèr LI. Pontryagin maximum principle in the theory of optimum systems. Avtomat i Telemekh. 1959;20:1320-1334. English Transl Automat Remote Control. 1959;20:1288-1302.

33. Sakawa Y, Shindo Y. On global convergence of an algorithm for optimal control. IEEE Trans Autom Control. 1980;25(6):1149-1153.

34. Shindo Y, Sakawa Y. Local convergence of an algorithm for solving optimal control problems. J Optim Theory Appl. 1985;46(3):265-293.

35. Varaiya P. N-person nonzero sum differential games with linear dynamics. SIAM Journal on Control. 1970;8(4):441-449.

Publisher's Note Springer Nature remains neutral with regard to jurisdictional claims in published maps and institutional affiliations. 